\title{
Intravenous hypertrophic Paccioni granulations: differentiation from venous dural thrombosis
}

\author{
P Giraud, S Thobois, M Hermier, E Broussolle, G Chazot
}

Paccioni granulations are normal anatomical structures that correspond to very enlarged arachnoid villi. ${ }^{1}$ They are involved in the filtration of CSF from the subarachnoid space to the venous system. Hypertropic Paccioni granulations (hPgs), usually secondary to high CSF pressure, can be symptomatic and responsible for lytic skull lesions or dural destruction. ${ }^{23}$ More exceptionally, the development of hPgs within the venous lumen makes their distinction from other pathological intravascular processes complicated. We report a case of unusual headache in a migrainous patient in whom hPgs in venous sinuses first led to an erroneous diagnosis of venous thrombophlebitis. This finding emphasises the importance of MRI as a mode of distinguishing between hPgs and dural venous thrombosis and suggests a possible link between hPgs and headache.

\section{Case report}

A 48 year old woman with a 25 year history of migraine without aura consulted for a recent unusual headache. She was treated with spironolactone and altizide for hypertension, and norprogesterone. Biological hyperthyroidism was also diagnosed due to toxic nodules and surgical treatment was planned. For 1 month, every night, she was woken at 2 00 am by severe, slightly pulsating, left sided
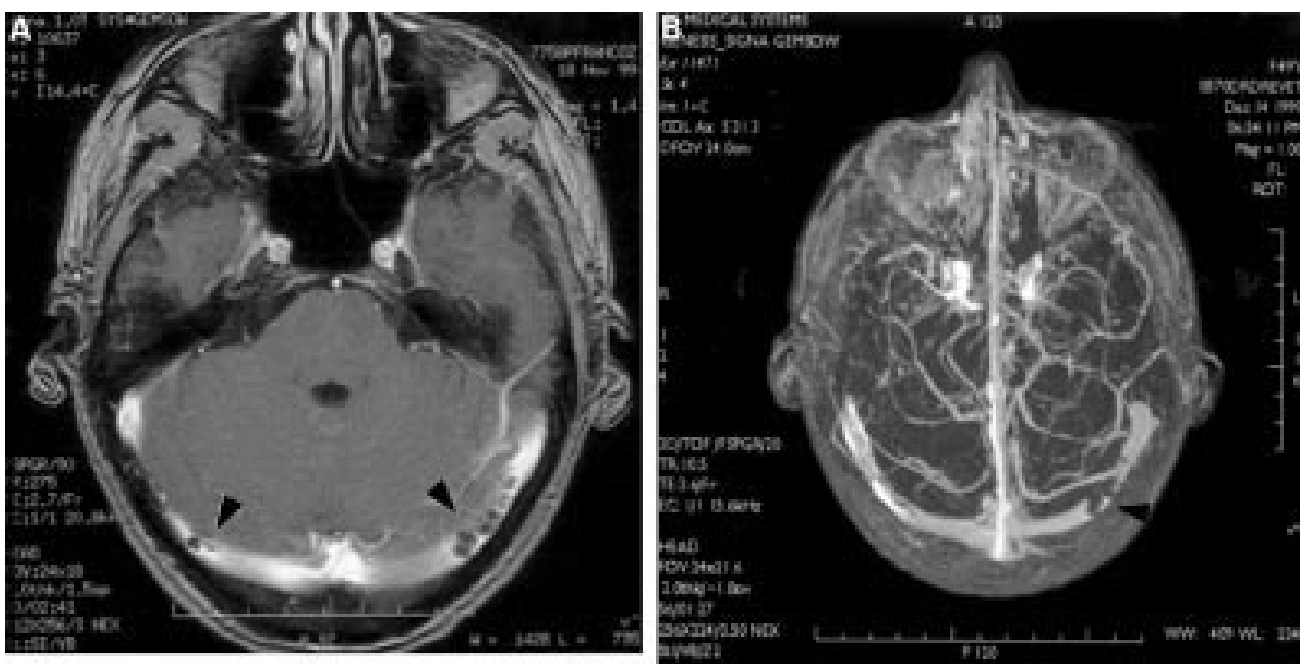

(A) Axial contrast: enhanced T1 weighted MR image (GEMSON-SYST, 1.0T, TR: 275; TE: 2.7) showing multiple hyposignal masses in right and left lateral sinuses (arrows). (B) Two dimensional time of flight $M R$ venogram (GEMSON-SYST, 1.0T, TR: 105; TE: 3.6) disclosing numerous focal defect images in the left dural lateral sinus (arrows). There is neither blood flow interruption nor increased size of small veins. hemicranial pain, sometimes associated with nausea without sonophobia. The headache was worsened by effort or by walking and lasted a few hours. Attacks were exclusively nocturnal. feine were ineffective. General and neurological examination proved normal. Axial brain MRI (GEMSON-SYS 1.0T) showed bilateral, round, clearly defined images within the left and right lateral venous sinuses. Intravascular images and hyperintense on the $\mathrm{T} 2$ weighted scans. No brain oedema or venous infarction was seen. No enhancement of the lesions was seen after gadolinium injection (figure A). A venous $M R$ angiogram showed only the image located in the left lateral sinus, which did not affect blood flow (figure B). Dural venous sinus thrombosis was diagnosed and continuous intravenous heparin infusion was administered. The headache was unchanged under therapeutic anticoagulation. Ocular fundi did not show papillary oedema. The CSF was clear with a normal opening pressure $\left(140 \mathrm{~mm} \mathrm{H}_{2} \mathrm{O}\right)$, cell counts (2 lymphocytes $/ \mu \mathrm{l})$, and protein concentration $(40 \mathrm{mg} / \mathrm{dl})$. The headache disappeared a few hours after lumbar puncture. Final diagnosis of hPg was made after further examination of MRI images. Heparin treatment was stopped and the patient left the unit with oral Treatment with paracetamol, codeine, or cafmasses were hypointense on $\mathrm{T} 1$ weighted
Service de Neurologie, Hôpital Neurologique Pierre Wertheimer, 59 Boulevard Pinel, 69003 Lyon, France

P Giraud

$S$ Thobois

G Chazot

Service de

Neuroradiologie

M Hermier

Correspondence to:

Dr P Giraud

Received 3 August 2000 and in final form

6 December 2000

Accepted 11 December 2000 
Summary of useful radiological data to distinguish between hypertropic Paccioni granulation and thrombus within dural venous sinuses ${ }^{4}$

\begin{tabular}{|c|c|c|}
\hline Dural venous with & Recent thrombosis & $h P g$ \\
\hline Aspect & Irregular, in entire segment of a sinus. & Round, regular well limited mass, impression in the skull \\
\hline Preferential topography & None & Lateral transverse sinus in the vicinity of venous entrance \\
\hline \multicolumn{3}{|r|}{ 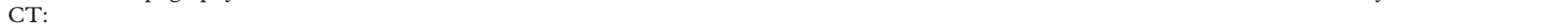 } \\
\hline Without contrast enhancement & Cord sign or spontaneous sinus hyperdensity. Indirect signs. & Never sinus hyperdensity. \\
\hline With contrast enhancement & $\begin{array}{l}\text { Delta sign or large defect within dural sinus. Abnormal } \\
\text { cortical vein opacification. Indirect signs. }\end{array}$ & $\begin{array}{l}\text { Hypodense or isodense relative to brain. Not contrast } \\
\text { enhancement of the mass. }\end{array}$ \\
\hline \multicolumn{3}{|c|}{ - } \\
\hline T1 weighted images & Early, isointense then hyperintense signal. Indirect signs. & Hypointense or isointense signal \\
\hline T2 weighted images & Early, hypointense the hyperintense signal. Indirect signs. & Hyperintense signal \\
\hline Signal varying with time of onset & Yes & Never \\
\hline Contrast enhanced $\mathrm{T} 1$ weighted images & Dural enhancement. indirect signs. & No or heterogeneous contrast enhancement. \\
\hline MR venogramm & $\begin{array}{l}\text { Absence of the flow distally to the occlusion. Abnormal } \\
\text { visualisation of collateral veins. }\end{array}$ & Normal flow distally and proximally to the mass. \\
\hline
\end{tabular}

Indirect radiological signs classically associated with venous thrombosis can be brain swelling, brain oedema, haemorrhagic stroke, visualisation of cortical veins or dural contrast enhancement. ${ }^{4}$

amytriptiline treatment (20 mg/day). Two months after discharge, nocturnal headache was absent and cerebral MRI was similar to previous scans.

\section{Discussion}

This case illustrates the possibility of mistaken diagnosis due to the presence of $\mathrm{hPgs}$ in the context of unusual headache in migrainous patients. The prevalence of $\mathrm{hPgs}$ in the general population is not currently known. Among 295 postmortem cerebral dural veins, Browder et al described the presence of two cases of hPgs in the superior sagittal sinus and 23 smaller hPgs in the lateral sinuses. In a retrospective radiological study, ${ }^{4} \mathrm{hPgs}$ were seen within the venous system in $24 \%$ of 573 contrast enhanced CT scans and in $13 \%$ of 100 brain MR images. However, radiological examination was not performed in non-symptomatic patients and, therefore, exact prevalence was not determined.

The presence of an experienced MRI radiologist reduces the risk of confusion between hPgs and thrombi or intravascular malignant masses. Radiological characteristics for distinguishing thrombosis from $\mathrm{hPgs}$ are summarised in the table. Among the signs that seem useful, hPg morphology seems particularly important; hPgs are classically round, clearly defined structures, preferentially localised in the lateral sinus in the vicinity of the venous entrance. ${ }^{4}$ These morphological characteristics on CT and MRI are so suggestive of $\mathrm{hPgs}$ that further radiological examination is unnecessary. ${ }^{14}$

Clinical symptoms of $\mathrm{hPg}$ have not been previously reported in detail. ${ }^{156}$ Browder et al postulated that they are asymptomatic, probably explained by their slow growth. Nevertheless, among symptoms regularly reported, recent or unusual headache is often described. In most patients, as in ours, headache was first thought to be symptomatic because of its unusual intensity, nocturnal appearance, consistent topography, and its resistance to analgesic drugs. ${ }^{7}$ One approach linking hPgs to headache is that they can induce symptomatic intracranial hypertension due to their effects on venous blood flow. ${ }^{1}$ However, even though rapid improvement after lumbar puncture is suggestive of such a mechanism, we noticed neither high CSF pressure nor papilloedema. It may be that hPgs have no role in the occurrence of headache. In fact, headache is a frequent complaint in the general population often leading to further investigation by radiological imagery that can show hPgs. ${ }^{8}$ Moreover, in our patient, as in the cases reported by Zagardo and Mamourian and Towfighi, spontaneous resolution of headache was seen without any action being taken on hPgs, equally suggesting that they are not involved in this affection.

In conclusion, hPgs have a particular aspect within the intravenous lumen contrasting with that of thrombi or malignant masses in the venous dural veins. Their radiological aspect is so particular that they can be easily diagnosed, making further imagery unnecessary. Further studies are required to improve our understanding of the relation between $\mathrm{hPg}$ and headache.

1 Mamourian AC, Towfighi J. MR of giant arachnoid granulation, a normal variant presenting as a mass within the dural venous sinus. AfNR Am f Neuroradiol 1995;16:9014 .

2 LeGross C. On the Pacchionan granulation. I Anat 1920;55:40-8.

3 Kuriowa T, Kajimoto Y, Ohta T, et al. Symptomatic hypertrophic Pacchionan granulation mimicking bone tumor: case report. Neurosurgery 1996;39:860-1.

4 Leach JL, Jones BV, Tomsick TA, et al. Normal appearance of arachnoid granulations on contrast-enhanced CT and MR of the brain: differentiation from dural sinus disease. AfNR Am F Neuroradiol 1996;17:1523-32.

5 Browder J, Browder A, Kaplan JJr. Benign tumor of the cerebral dural sinuses. $\mathcal{F}$ Neurosurg 1972;37:576-9.

6 Zagardo MT. Proeminent Paccioni granulations. AfNR Am f Neuroradiol 1997;18:1594-5.

7 Lance JW, Goasby PJ. The investigation and general management of headache. In : Lance JW, Goasby PJ, eds. Mechanism and management of headache. 6th ed. Cambridge: Butterworth Heinemann, 1998:291-8. 